\title{
27 - Pituitary hormone changes in adolescents with overweight/obesity under a multidisciplinary treatment programme: preliminary results of the evasion study
}

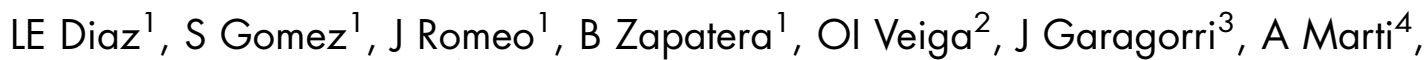 \\ C Campoy, G Morande ${ }^{6}$ and A Marcos ${ }^{1}$
}

${ }^{1}$ Immunonutrition Research Group, Department of Metabolism and Nutrition, Institute of Food Science, Technology and Nutrition (ICTAN), Instituto del Frio, Spanish National Research Council (CSIC), Madrid, Spain: ${ }^{2}$ Department of Physical Education, Sport and Human Movement, Facultad de Formacion del Profesorado y Educacion, Universidad Autonoma de Madrid, Madrid, Spain: ${ }^{3}$ School of Health Sciences, University of Zaragoza, Zaragoza, Spain: ${ }^{4}$ Department of Physiology and Nutrition, University of Navarra, Pamplona, Spain: ${ }^{5}$ Department of Pediatrics, University of Granada, Granada, Spain: ${ }^{6}$ Department of Psychiatry, Hospital Infantil Universitario Nino Jesus, Madrid, Spain

Introduction: Numerous endocrine abnormalities are associated with obesity. The present study was aimed to evaluate the effects after 2 and 13 months of a multidisciplinary obesity treatment programme, called EVASYON, on pituitary hormone levels in adolescents with overweight/obesity.

Method: Thirty-five adolescents (16 girls/19 boys; mean BMI $31 \cdot 56(\mathrm{SD} 4 \cdot 70) \mathrm{kg} / \mathrm{m}^{2}$; range: $25 \cdot 01-41 \cdot 23$; mean age $14 \cdot 08$ (SD $1 \cdot 11$ ) years; range: $12 \cdot 0-16 \cdot 0$ ) from Madrid were classified within the overweight/obesity ranges according to the International Obesity Task Force BMI criteria, and were voluntarily recruited into a longitudinal multidisciplinary intervention study based on calorie-restricted diet (10-40\%), increased physical activity (calorie expenditure $=15-23 \mathrm{kcal} /$ kg body weight/week), psychological therapy and nutritional education for 13 months. The treatment period consisted of two phases: (i) intensive during the first 2 months (2 mo) and (ii) extensive during the consecutive 11 months (13 mo).
This study only includes some preliminary data from one city and related to both intervention periods of the EVASYON programme. Pituitary hormones: follicle-stimulating hormone (FSH), luteinizing hormone (LH), thyrotropin hormone (TSH), growth hormone (GH), prolactin (PRL) and adrenocorticotropic hormone (ACTH) levels were measured before and after both intervention periods.

Results: PRL serum levels $(P<0 \cdot 05)$ and BMI (5.54\%) decreased after 2 mo. After $13 \mathrm{mo}$ FSH and TSH serum levels $(P<0 \cdot 05)$ decreased and a tendency to decrease PRL serum levels together with decreased BMI $(-7 \cdot 35 \%)$ values were shown. A positive correlation between PRL levels and BMI after 2 mo $(r=0.333 ; P<0 \cdot 05)$ was found.

Conclusions: These results suggest that weight reduction in adolescents with overweight/obesity under the EVASYON intervention programme can improve the endocrine disturbances associated with obesity.

\section{8 - Insulin resistance among overweight/obese pre- and post-pubertal paediatric patients}

\author{
A Piedimonte, A Mosca, AM Caiazzo, D Guttadoro, A Cafarotti, CM L'Amante, \\ R Mercurio, RE Papa and A Vania \\ Centro di Dietologia e Nutrizione Pediatrica, I Facoltà di Medicina e Chirurgia, 'Sapienza' Università di Roma, Italy
}

\begin{abstract}
Aim: To verify the possible relationship of insulin resistance (IR) with pubertal stage.

Introduction: Scientific literature outlines that insulin sensitivity changes throughout the puberty. Obese females (F) show higher IR rates than males (M); different changes in fat mass, usually happening in puberty also among these patients, are generally claimed as an explanation.
\end{abstract}

Method: 755 overweight/obese patients (Ow/OB; F 398, M 357; average age $=10 \cdot 25$ (sD 2.84) years $)$ were considered. OW/OB was divided in two subgroups: pre-pubertal (PRE, Tanner's stage 1-3; F 219, $M$ 227) and post-pubertal (POST, Tanner's 4 and 5; F 179, M 130). Statistical analysis used Student's $t$ and $\chi^{2}$ tests. 
Results: Waist/height ratio $(\mathrm{W} / \mathrm{h})$ is greater in $\mathrm{M}$ general sample $(P=0 \cdot 01)$ but also in POST-F:PRE-F $(P=0 \cdot 05)$ and in PRE-M:PRE-F $(P=0 \cdot 0001)$. At large, blood glucose was higher in $\mathrm{M}$, independently from puberty $(P=0 \cdot 01)$, while insulin was similar in $\mathrm{F} / \mathrm{M}$. After sub-grouping, insulin was higher in post-F/M (both $P=0 \cdot 0001) v$. PRE, while glucose was higher in POST-F:POST-M $(P=0 \cdot 01)$.

Similar behaviour for insulin resistance-homeostasis model assessment (IR-HOMA): higher in POST-F/M $v$. PRE (both $P=0 \cdot 0001$ ). Besides, IR-HOMA $>2.5$ risk is higher in POST (whole sample, F, M), but POST-M have a greater risk $(\mathrm{OR}=2 \cdot 11$ POST:PRE, $P=0 \cdot 0001 ; \mathrm{OR}=2 \cdot 45$ POST-M:PRE-M, $\quad P=0 \cdot 02 ; \quad$ OR $=1.94 \quad$ POST-F:PRE-F, $P=0 \cdot 01)$.

Conclusions: $\mathrm{M}$ attending our outpatients service seems in poorer nutritional (higher $\mathrm{W} / \mathrm{h}$ ) and metabolic conditions (higher pathologic IR-HOMA risk) than F, with a slight indication that abdominal fat distribution might not be the only explanation for IR appearance: other factors should be considered and studied.

\title{
29 - Insulin resistance risk among ex-preterm overweight/obese patients
}

\author{
A Piedimonte, A Mosca, AM Caiazzo, D Guttadoro, A Cafarotti, CM L'Amante, R Mercurio, \\ RE Papa and A Vania
}

Centro di Dietologia e Nutrizione Pediatrica, I Facoltà di Medicina e Chirurgia, 'Sapienza' Università di Roma, Italy

Introduction: According to the 'thrifty phenotype' hypothesis, ex-preterm (ExP) children, if overfed in infancy, show a greater insulin resistance (IR) risk than AGA (appropriate gestational age) children. ExP also shows a larger waist circumference (W), due to greater extent of abdominal fat: this might trigger off IR.

Method: 655 valid overweight/obese patients (F 356, M 309 ; average age $=10 \cdot 43(\mathrm{SD} 2 \cdot 84)$ years) were considered: ExP 118 (F 62, M 56), AGA 547 (F 294, M 253). Anthropometric indexes studied were $\mathrm{W}$ and waist/height ratio (W/h). Insulin resistance-homeostasis model assessment (IR-HOMA), studied in 569 patients (ExP 102: F 54, M 48; AGA 467: F 254, M 213), led to sub-grouping them in: IR-HOMA >2.5 (ExP 53: F 26, M 27; AGA 226:
F 129, M 97) and IR-HOMA <2.5 (ExP 49: F 28, M 21; AGA 241: F 125, M 116). Statistical analysis used Student's $t$ and $\chi^{2}$ tests.

Results: W was $>95$ thC in $97 \cdot 4 \%$ of $\operatorname{ExP} v \cdot 91 \cdot 4 \%$ of AGA; W/h was pathologic $(>0.5)$ in $92.4 \%$ of $\operatorname{ExP} v$. $89 \cdot 0 \%$ of AGA. ExP have a higher risk of $\mathrm{W}>95$ thC and $\mathrm{W} / \mathrm{h}>0.5$ than AGA (W OR: $\mathrm{F}=5 \cdot 33, \mathrm{M}=2 \cdot 23$; W/h $\mathrm{OR}=1.5$ in both $\mathrm{F} / \mathrm{M}$, respectively). ExP also have IRHOMA $>2.5$ more frequently, with higher risk for M (OR: $\mathrm{F}=0 \cdot 9 ; \mathrm{M}=1 \cdot 5$ ).

Conclusions: In our experience, ExP of both genders show a greater extent of $\mathrm{W}>95^{\circ} \mathrm{C}$ and $\mathrm{W} / \mathrm{h}>0.5$ than AGA, but an IR risk just slightly higher $(\mathrm{OR}=1 \cdot 25)$. $\mathrm{M}$ ExP seems to be at higher risk than F: literature lacks of data about this point.

\section{0 - Relationship among insulin resistance, blood lipids and blood pressure in a population of paediatric overweight/obese patients}

\author{
A Mosca, A Piedimonte, AM Caiazzo, RE Papa, D Guttadoro, A Grimaldi, \\ S Zorkadis, A Vania \\ Centro di Dietologia e Nutrizione Pediatrica, I Facoltà di Medicina e Chirurgia, 'Sapienza' Università di Roma, Italy
}

Introduction: Within the ample debate about Metabolic Syndrome, its primum movens and its pathophysiology, a relationship among high insulin resistance-homeostasis model assessment (IR-HOMA), blood lipids (BL: triglycerides 